\title{
El Voluntariado juvenil en América del Sur: Un análisis de su orientación y formalización utilizando la teoría de los orígenes sociales de la sociedad civil'
}

\section{Youth Volunteerism in South America: An analysis of its orientation and formalization using the theory of social origins of civil society}

\section{PhD. René Olate}

René Olate es Ph.D. in Social Work, Master of Social Work, Licenciado en Ciencias Sociales y Asistente Social. Actualmente se desempeña como académico de Boston College Graduate School of Social Work. Su dirección postal es McGuinn Hall, 209140 Commonwealth Avenue Chestnut Hill, MA 02467 USA. Su e-mail es: olate@bc.edu

\begin{abstract}
Resumen
Los programas de voluntariado juvenil se han destacado por su crecimiento y contribución a mejorar los desafíos sociales, económicos, políticos y medioambientales. Usando una muestra de programas de voluntariado juvenil de cinco paises de América del sur $(n=304)$ y comparándola con una muestra de paises de América Latina $(n=533)$, este documento analiza la orientación y formalización de estos programas utilizando la teoría de los orígenes sociales de la sociedad civil. Se emplea la metodología de "path análisis" (análisis de senderos) para identificar los factores asociados a la orientación y formalización del voluntariado juvenil en el nivel de programa, de organización y de pais. El análisis de los datos indica que las relaciones entre la variable "orientación del voluntariado" y las variables multi-niveles no son estadisticamente significativas para ambas regiones. Las relaciones entre las variables "formalización del voluntariado" y "efectividad del gobierno" y "nivel de pobreza" (a nivel nacional), y el "liderazgo juvenil" y los "programas incluyentes" (a nivel de programa) son estadisticamente significativas para América Latina. Para América del Sur solo las variables a nivel de programa son significativas. Por tanto, el análisis ayuda a confirmar parcialmente algunos de los postulados de la teoría de los orígenes sociales de la sociedad civil. Finalmente, se presentan algunas sugerencias para la intervención social.
\end{abstract}

Palabras claves. voluntariado, jóvenes, América Latina, formalización, sociedad civil.

\begin{abstract}
Youth volunteer programs have been highlighted for their growth and contribution to the alleviation of social, economic, political, and environmental challenges. Employing a sample of youth volunteer programs from five countries in South America $(n=304)$, in comparison with a sample of Latin America and the Caribbean $(n=533)$, this paper analyzes the orientation and formalization of these programs using the theory of social origins of civil society. A path analysis model is employed to identify factors associated with the orientation and formalization of youth volunteer programs at the program, organization, and national levels. The analysis indicates that the relationships between the variable "orientation" and the multi-level indicator are not statistically significant for both models. The variables "effectiveness of government" and "level of poverty" (at the national level), and "leadership" and "inclusiveness" (at the program level) are statistically significant when associated with the "level of formalization" for Latin America. For South America only, the variables at program level are significant. Thus, the analyses partially confirm the claims of the theory of social origins of civil society. Finally, some recommendations for social intervention are presented.
\end{abstract}

Keywords. volunteerism, youth, Latin America, formalization, civil society.

1 El autor agradece al Centro para el Desarrollo Social de Washington University por la utilización de la base de datos del estudio "Servicio Voluntario Juvenil en América Latina y el Caribe: Un estudio regional (2006-2007)". El autor desarrolló su tesis doctoral como uno de los investigadores de este proyecto en el cual tuvo la responsabilidad de desarrollar el cuestionario y analizar los datos. 


\section{Introducción}

Las organizaciones y programas de voluntariado se han destacado en las últimas décadas por su importante crecimiento y contribución a los importantes desafíos económicos, sociales, políticos y medioambientales que experimenta la región (CIVICUS, IAVE, \& UNV, 2008; Naciones Unidas, 2005). Las Naciones Unidas dan un decisivo apoyo a las estrategias de voluntariado al declarar el año 2001 como el Año Internacional del Voluntariado. Además, han identificado el importante rol que puede jugar el voluntariado en el logro de sociedades más cohesionadas, con mayores niveles de confianza e integración social (United Nations, 2008a) y en la consecución de las Metas de Desarrollo del Milenio (United Nations, 2008b).

En un contexto de recursos limitados, los programas de voluntariado pueden entregar un aporte significativo en diversas estrategias de combate a la pobreza y desarrollo económico y social (United Nations, 2005b). Se observa que existe un mayor apoyo a los programas de voluntariado, especialmente juvenil, desde las organizaciones de la sociedad civil, los gobiernos nacionales, regionales y locales y los organismos internacionales (Olate, 2007). Este apoyo se ha manifestado en el aumento del financiamiento de las organizaciones y programas de voluntariado (UNV, 2001; United Nations, 2005a). Esta mayor disponibilidad de recursos potencialmente influenciará la orientación y generará un mayor nivel de formalización de estas organizaciones y programas.

Los programas de voluntariado no son nuevos (Landim, 2001), están enraizados en la historia y cultura de los países, lo que constituye una novedad son los procesos de institucionalización, formalización, profesionalización, desarrollo y expansión de estas organizaciones (Olate, 2007; Olate, Johnson, \& McBride, 2007). El voluntariado ha sido tradicionalmente estudiado desde una perspectiva individual (motivaciones, preferencias, percepciones, valores, etc.) (Wilson, 2000) incluso en los estudios comparativos internacionales la unidad de análisis son los individuos (Inglehart, 2003). Esta investigación analiza los programas de voluntariado juvenil en América del sur utilizando la teoría de los orígenes sociales de la sociedad civil (Salamon \& Anheier, 1998) y los conceptos de orientación y formalización del voluntariado.

El objetivo de este documento es analizar la relación existente entre la orientación y formalización de los programas de voluntariado juvenil y un conjunto de variables a nivel de programa, organización y país. Este estudio utiliza la base de datos del estudio de- sarrollado por el Centro para el Desarrollo Social de Washington University titulado "Servicio Voluntario Juvenil en América Latina y el Caribe: Un estudio regional (2006-2007)" (McBride, Olate, \& Johnson, 2008). La base de datos incluye 304 programas de voluntariado juvenil (223 organizaciones) de Argentina, Brasil, Colombia, Perú y Venezuela. Además, se utilizan los datos del Banco Mundial (World Bank, 2007) y de la Comisión Económica para América Latina (ECLAC, 2007) para analizar las variables del nivel nacional incluidas en el modelo. Se utiliza la metodología de "path análisis" (análisis de senderos) para identificar los factores asociados a la orientación y formalización del voluntariado juvenil en los niveles nacional, de organización y de programa. Para el cumplimiento de este objetivo, se desarrolla en la primera parte los aspectos conceptuales, en la segunda parte se presenta el modelo conceptual y las hipótesis de investigación, en la tercera parte se abordan los aspectos metodológicos, en la cuarte parte se presentan los resultados para finalmente destacar algunas conclusiones y comentarios finales.

\section{Antecedentes conceptuales}

Voluntariado. El concepto de voluntariado tiene una pluralidad de significados y una multiplicidad de manifestaciones (García, 1994), el cual varía de acuerdo a los diferentes períodos históricos, niveles de desarrollo, sistemas políticos, culturas y religiones (Cnaan, Handy, \& Wadsworth, 1996; Handy, Cnaan, Brudney, Ascoli, Meijs, \& Ranade, 2000; Landim, 2001, 2005). Diversos autores sugieren que en la década de 1960 y 1970, debido a factores del contexto político, social y económico de la región, surge una nueva forma de voluntariado mayoritariamente juvenil denominada voluntariado "militante" o "transformador" (Bettoni \& Cruz, 2002; Jaramillo, 1993). Entre los factores que influencian el surgimiento de este nuevo tipo de voluntariado se encuentran: la revolución cubana y las ideologías socialistas, la reacción ante los regímenes autoritarios, la teología de la liberación y el número creciente de organizaciones no gubernamentales.

Este nuevo voluntariado rechaza las formas tradicionales de voluntariado y sus vínculos con la elite, la caridad y el asistencialismo, proponiendo un modelo orientado a la solidaridad, el desarrollo y las transformaciones sociales (Thompson \& Toro, 1999; Bettoni \& Cruz, 2002). Mientras el voluntariado tradicional o conservador tiene motivaciones principalmente religiosas, el nuevo voluntariado militante se caracteriza por tener motivaciones 
políticas y por generar fuertes alianzas con los movimientos sociales.

Entre la década de 1960 y 1980, la mayoría de los países de América Latina tuvieron gobiernos dictatoriales, los cuales impusieron restricciones a la participación ciudadana organizada y con ello a algunas iniciativas de voluntariado. Muchos de estos gobiernos consideraban que el voluntariado estaba fuertemente asociado a los movimientos sociales insurgentes. A pesar de ello, los programas de voluntariado continuaron realizando sus actividades, pudiéndose distinguir dos grandes tipos: los "oficialistas" y los "alternativos" (Thompson $\&$ Toro, 1999). El "voluntariado oficialista" corresponde a un tipo de voluntariado tradicional, centrado en la asistencia social, que fue promovido por los gobiernos autoritarios a través de las esposas de los militares y de institutos de la juventud. Mientras que el "voluntariado alternativo" estaba vinculado a los diversos movimientos sociales que buscaban recuperar la democracia y defender los derechos humanos, entre ellos: los comedores solidarios, talleres laborales, grupos juveniles, grupos de salud y comités sin casas (FLACSO-MORI-CERC, 2002).

Otros autores también denominan al "voluntariado alternativo" como "voluntariado social", el cual surge al interior de grupos católicos influenciados por la teología de la liberación y las metodologías de educación popular. Estas formas de acción voluntaria surgen también como una reacción al voluntariado tradicional impulsado por la Iglesia, al cual se le criticaba la promoción del asistencialismo, ser el hobby de los ricos, y su enfoque en problemas aislados sin atender a las causas de los mismos. De acuerdo a Jaramillo (1993), el voluntariado social requiere sinceridad y compromiso por parte del voluntario para lograr una verdadera inmersión en las vidas de aquellos a los que pretende servir.

Ante la fuerte presión de los movimientos sociales, los gobiernos autoritarios disminuyen las restricciones sobre la participación ciudadana dando inicio a los procesos de transición democrática. Los procesos de democratización, asociados a la apertura de las economías y a la tendencia creciente a la globalización facilitaron la entrada de organizaciones internacionales de voluntariado y de programas de servicio de universidades de Europa y Estados Unidos. Estos programas y organizaciones tienen objetivos diversos, entre los cuales se destaca la promoción de sociedades civiles activas a través de una mayor responsabilidad social y participación cívica (Toro \& Moret, 2000).

En la década de los noventa, como reacción a los agudos problemas sociales, económicos y ambientales, surgen diversos tipos de organizaciones y programas de voluntariado en la región, con orientaciones plurales, muchos de ellos comprometidos con la protección y preservación del medio ambiente (Butcher, 2008). También emerge una perspectiva de estudio del voluntariado menos abordada para referirse a este fenómeno: la dimensión simbólica y cultural (Landim, 2001), la cual tiene un rol central en la reproducción de los valores e imaginarios sociales de cada país y de la región en general.

Comenzando el siglo XXI, se observa en la región una pluralidad de formas de voluntariado con diferentes motivaciones y finalidades, que tienen sus raíces en los tipos anteriormente descritos. En las organizaciones de voluntariado juvenil conviven formas de voluntariado tradicional, caracterizados por su énfasis en la caridad y el asistencialismo, y el militante, "nuevo voluntariado" (Landim, 2005) o voluntariado de la promoción (García, 1994), con su fuerte acento en valores de transformación social y justicia social. Además de existir múltiples maneras de referirse a este fenómeno (voluntariado, trabajo voluntario, voluntariado social, acción voluntaria y servicio voluntario) (OIJ, 2002), también están los nuevos y tradicionales temas asociados al voluntariado: formas de capital social, responsabilidad social y filantropía. Esta pluralidad también se ve reflejada en las distintas entidades que actualmente promueven y financian el voluntariado: el estado, las empresas, organizaciones nacionales del tercer sector y organismos internacionales.

Las definiciones de voluntariado son diversas y destacan diferentes dimensiones del concepto (Handy et al., 2000; Landim, 2001; Roitter, 2002) reflejando, de alguna manera, la pluralidad de ideologías y organizaciones en las cuales se desarrolla. Una definición de voluntariado bastante aceptada, pero no por ello exenta de controversia, es planteada por Brown (1999): la entrega de un servicio o el desarrollo de un trabajo sin recibir un pago monetario. De un modo similar, la Cruz Roja define a los voluntarios como aquellos individuos que desarrollan una actividad, no vinculada a su trabajo asalariado o a sus responsabilidades normales, contribuyendo con tiempo y servicio a una causa no lucrativa (Smith, 1989). Estas definiciones recogen uno de los elementos centrales que caracterizan al voluntariado, la idea de ausencia de salario. Sin embargo, esta concepción será puesta en discusión a través del concepto de la acción voluntaria en el contexto de los procesos de institucionalización, formalización y profesionalización del voluntariado. 
La acción voluntaria se expresa en diversas organizaciones: sindicales, gremiales, culturales, deportivas, recreativas, educacionales (en sus diversos niveles y tipos de enseñanza), y religiosas (católicas, protestantes, judías, etc.). Además, está vinculada a las actividades y temáticas de los tradicionales y nuevos movimientos sociales (etnias, género, ecología, etc.). Las organizaciones y programas en los cuales se desarrolla el voluntariado son tanto formales, amparadas bajo distintas figuras legales, como informales, tales como organizaciones de mujeres, jóvenes y de la tercera edad (FLACSO-MORI-CERC, 2002). Esta heterogeneidad de manifestaciones del voluntariado genera una serie de dificultades al intentar conceptualizar el tema.

En este documento, el concepto de acción voluntaria se entiende a lo largo de un continuum (ver Figura No. 1) que se distingue por varias dimensiones tales como el nivel de estructura, formalidad y compromiso de tiempo (McBride \& Sherraden, 2007). En un extremo del continuum se encuentran las actividades solidarias informales de ayuda mutua, de atención y cuidado entre los miembros de una comunidad. En el punto intermedio se encuentran las actividades de voluntariado convencionales, mientras que en el otro extremo del continuum se encuentran modelos más institucionalizados y formales de voluntariado, los cuales se denominan servicio cívico o servicio voluntario. Sherraden (2001a) define el servicio cívico como "un período organizado de compromiso sustancial y contribución a la comunidad mundial, nacional y local, reconocido y valorado por la sociedad, con una compensación monetaria mínima para el participante [o sin ella]" (p. 2).

FIGURA $N^{\circ} 1$.

\section{CONTINUUM DE LA ACCIÓN VOLUNTARIA}

\begin{tabular}{ccc} 
Informal & Voluntariado & Servicio \\
$\begin{array}{c}\text { Expresiones } \\
\text { informales de } \\
\text { ayuda mutua }\end{array}$ & $\begin{array}{c}\text { convencional } \\
\text { voluntario } \\
\text { o cívico }\end{array}$ \\
\hline
\end{tabular}

Fuente: Basado en McBride \& Sherraden (2007)

A continuación se presentan ejemplos para cada uno de las formas de acción voluntaria. Las actividades de ayuda mutua representan una manera informal de cooperación entre los miembros de una comunidad. Constituyen el eje de la asociación grupal primaria de familias y comunidades y se caracte- rizan por niveles relativamente bajos de estructura, formalidad y compromiso de tiempo. Un ejemplo de esta forma de acción voluntaria son las actividades de apoyo y cooperación de las comunidades ante desastres naturales tales como terremotos, desplazamientos de tierra, inundaciones e incendios. Este tipo de cooperación no está estructurada en organizaciones formales sino que responde a normas sociales y a valores enraizados en la cultura de las comunidades.

Al avanzar a lo largo del continuum encontramos las actividades de cooperación algo más formales y estructuradas, las cuales corresponden a organizaciones y programas de voluntariado convencionales o clásicos en la región. Esta forma de voluntariado, preponderante en la región, corresponde a actividades que se dan al interior de una organización o programa pero que tienen una periodicidad y duración limitada. Por ejemplo, la entrega de botiquines de emergencia o un programa de tutoría escolar son actividades que se llevan a cabo en un sólo día o en un fin de semana. Las experiencias de aprendizaje-servicio realizadas por estudiantes de enseñanza primaria y secundaria corresponden a este tipo de voluntariado. Los programas de servicio voluntario o cívico se caracterizan por poseer una estructura, son formales y el compromiso de tiempo de los voluntarios es intensivo y extenso en el tiempo. Los programas "Opción Colombia", "Servicio País" de Chile, y "Servicio Social" de México son ejemplos de este tipo de programas en la región. El ejemplo norteamericano clásico de estos programas es el "Peace Corps", en el cual los jóvenes se comprometen a servir en un país por un período de tiempo por el cual reciben una compensación mínima.

Para clarificar la idea de continuum de la acción voluntaria, las dimensiones de voluntariado desarrolladas por Smith (1999) son muy útiles (ver Cuadro No. 1). Smith (1999) en el documento de trabajo preparado para la reunión de expertos en voluntariado y desarrollo social de las Naciones Unidas identifica cinco dimensiones del voluntariado: a) recompensa económica, b) libre voluntad, c) naturaleza del beneficio, d) entorno organizacional y e) nivel de compromiso. La idea de recompensa o retribución económica apunta al debate en torno a si los voluntarios deben o no recibir algún tipo de compensación por la actividad que realizan. La idea de libre voluntad se refiere a la ausencia de obligación para participar en las actividades o prestar el servicio. La naturaleza del beneficio se refiere a la necesidad de que exista un beneficiario que sea distinto del voluntario, de manera que no sea el 
voluntario el único que se beneficie de las actividades. El entorno organizacional se refiere a que la actividad voluntaria se desarrolla desde un tipo de organización formal o informal. Finalmente, el nivel de compromiso se refiere a la periodicidad e intensidad de las actividades voluntarias, el debate apunta a establecer si las actividades esporádicas constituyen voluntariado.

A diferencia de otras expresiones de la acción voluntaria, el servicio voluntario o cívico puede o no ser voluntario. En general, los participantes en los programas de servicio voluntario reciben alguna forma de compensación monetaria o en bienes y servicios, sirven a una población o grupo específico pero también se benefician ellos mismos a través de su formación, tienen un compromiso de tiempo mayor e intensivo y su participación se da dentro de un contexto organizacional formal. Es conveniente señalar que al interior de una misma organización se pueden encontrar formas de acción voluntaria esporádica, que corresponderían a programas de voluntariado de tipo convencional, y programas de servicio voluntario caracterizados por su mayor nivel de estructura y formalización. Por ejemplo, "Opción Colombia" y un "Techo para Chile" desarrollan programas y actividades con distintos niveles de compromiso de tiempo y que tienen como denominador común un alto grado de formalización de sus estructuras organizativas (Olate, Johnson, \& McBride, 2006)

En este sentido, se puede señalar que organizaciones de voluntariado en la región ofrecen distintos tipos de programas y actividades voluntarias a los jóvenes con distintos niveles de compromiso, responsabilidad y remuneración. Por tanto, en una misma organización de voluntariado podemos encontrar una oferta de la acción voluntaria que recorre todo el continuum. Por ejemplo, en un "Techo para Chile" o en "Opción Colombia" se pueden encontrar programas de voluntariado que desarrollan exclusivamente actividades puntuales, asemejándose a un tipo de expresión de la acción voluntaria más informal, programas de voluntariado más convencionales en los cuales las dimensiones de la acción voluntaria se encuentran en una etapa intermedia del continuum, y finalmente, programas que son más estructurados, similares a los modelos de servicio voluntario o cívico en los cuales los participantes reciben una remuneración o incentivos económicos.

El continuum de la acción voluntaria puede presentar algún grado de controversia en la región, especialmente en su polo más institucionalizado que corresponde al servicio voluntario o cívico. Algunos investigadores y profesionales vinculados al tema del voluntariado no lo consideran un tipo de acción voluntaria debido a que puede incluir algún tipo de remuneración. Sin embargo, se considera que en los procesos de institucionalización, formalización y profesionalización de los programas de voluntariado, la remuneración o apoyo económico surge como un elemento necesario en la gestión y desarrollo de los programas. Las cinco dimensiones del voluntariado ayudan a entender la complejidad que encierra esta actividad y las dificultades para alcanzar una definición única de voluntariado. Como

CUADRO NO 1

DIMENSIONES DEL VOLUNTARIADO

\begin{tabular}{|c|c|c|c|}
\hline & $\begin{array}{c}\text { Expresiones Informales } \\
\text { de Ayuda Mutua }\end{array}$ & $\begin{array}{l}\text { Voluntariado } \\
\text { Convencional }\end{array}$ & $\begin{array}{c}\text { Servicio Voluntario } \\
\text { o Cívico }\end{array}$ \\
\hline $\begin{array}{l}\text { Recompensa } \\
\text { Económica }\end{array}$ & No & No & $\mathrm{Si}$ \\
\hline Libre Voluntad & Voluntario & Voluntario & Voluntario/ Obligatorio \\
\hline \multirow{2}{*}{$\begin{array}{l}\text { Naturaleza del } \\
\text { Beneficio }\end{array}$} & Comunidades & Comunidades & Comunidades / Voluntarios \\
\hline & $\begin{array}{l}\text { Asociaciones } \\
\text { Informales }\end{array}$ & $\begin{array}{l}\text { Organizaciones Formales } \\
\text { e Informales }\end{array}$ & Organizaciones Formales \\
\hline $\begin{array}{l}\text { Entorno } \\
\text { Organizacional }\end{array}$ & Ocasional & $\begin{array}{l}\text { Ocasional y Corto } \\
\text { Periodo de Tiempo }\end{array}$ & $\begin{array}{l}\text { Intensivo y Largo } \\
\text { Periodo de Tiempo }\end{array}$ \\
\hline $\begin{array}{l}\text { Nivel de } \\
\text { Compromiso }\end{array}$ & & & \\
\hline
\end{tabular}

Fuente: el autor 
se mencionó anteriormente, el voluntariado tiene diversos significados y toma distintas formas de acuerdo a las diferentes culturas y contextos institucionales.

Organizaciones de voluntariado. Además del continuum de la acción voluntaria, otra conceptualización complementaria para entender el voluntariado es la de organizaciones de voluntariado híbridas con múltiples propósitos (Hasenfeld \& Gidron, 2005). De acuerdo a Hasenfeld y Gidron (2005) estas organizaciones poseen cuatro características interrelacionadas: a) tienen como misión la promoción de valores culturales que son muchas veces disonantes con los valores dominantes en la sociedad, b) entregan distintos tipos de servicios a través de los cuales modelan y promueven el cambio social; c) tienen como foco la promoción de una identidad colectiva, y d) poseen múltiples propósitos que incluyen la promoción de valores orientados al cambio social, la entrega de servicios y la ayuda mutua.

Hasenfeld y Gidron (2005) señalan que estas organizaciones necesitan ser entendidas en el contexto de tres teorías: de la sociedad civil, de los movimientos sociales y de las organizaciones sin fines de lucro. La utilización de esta perspectiva conceptual, ayuda a entender y explicar más comprehensivamente la emergencia, la movilización de recursos y el compromiso de las organizaciones de voluntariado. La perspectiva de estos autores es importante, sin embargo, las teorías de las organizaciones sin fines de lucro pueden entenderse como parte de las teorías de la sociedad civil. Por tanto, dos extensos y variados cuerpos teóricos pueden ayudar a contextualizar los programas de voluntariado en la región: las teorías de la sociedad civil y las teorías de los movimientos sociales. Las posibilidades de conectar ambas cuerpos teóricos son múltiples (CIVICUS, IAVE, \& UNV, 2008; Veltmeyer, 2004) y ofrecen un camino fructífero de reflexión analítica y empírica.
Por ejemplo, las organizaciones de voluntariado con una orientación sociopolítica pueden ser entendidas como expresiones de la sociedad civil y como manifestaciones de nuevos y tradicionales movimientos sociales.

Sociedad civil. En este estudio se privilegia la perspectiva conceptual de la sociedad civil, la cual puede ser entendida como la acción colectiva voluntaria basada en intereses, objetivos y valores compartidos, la cual excluye las relaciones de mercado, de familia o del estado. La sociedad civil también se denominada sector sin fines de lucro, no gubernamental, voluntario, independiente o tercer sector. Una de las perspectivas de la sociedad civil que más destaca el rol de las organizaciones de voluntariado es la teoría de los orígenes sociales de la sociedad civil (Salamon \& Anheier, 1998; Salamon, Sokolowski, \& Anheier, 2000). Esta teoría ha sido utilizada para analizar y comparar variaciones en el tamaño y composición de las organizaciones de voluntariado en más de 40 países.

Para analizar las organizaciones del tercer sector, Salamon y Anheier desarrollan la Clasificación Internacional de las Organizaciones sin fines de lucro (ICNPO, por sus siglas en inglés) (Salamon, et al., 2004). De acuerdo a esta clasificación, las organizaciones pueden ubicarse en 14 categorías, utilizando como criterio la actividad principal de la organización. Utilizando esta clasificación Salamon y sus asociados reagrupan a las organizaciones en dos categorías: a) organizaciones orientadas al servicio y b) organizaciones orientación a la expresión de necesidades (Salamon \& Anheier, 1992; 1996) (Ver Cuadro $N^{\circ} 2$ ). Las organizaciones orientadas al servicio buscan asistir a las personas en sus necesidades más básicas, mientras las orientadas a la expresión concentran sus actividades y objetivos en torno a la cultura, la estética y la promoción de valores políticos.

CUADRO 2

TIPO DE ORGANIZACIÓN

\begin{tabular}{|l|l|}
\hline Servicio & Expresión \\
\hline a) Educación & a) Cultura, recreación y artes \\
\hline b) Salud & b) Medio ambiente y protección de animal \\
\hline c) Servicios a los niños, jóvenes y familias & c) Derecho e incidencia en la política \\
\hline d) Desarrollo comunitario & d) Filantropía \\
\hline e) Vivienda & e) promoción del Voluntariado \\
\hline f) Empleo y capacitación & f) Religión \\
\hline g) Emergencia y asistencia en desastres & g) Asociación de profesionales y de negocios, y sindicatos \\
\hline
\end{tabular}


Salamon y Anheier prueban satisfactoriamente la teoría de los orígenes sociales de la sociedad civil al explicar los patrones de desarrollo de las organizaciones sin fines de lucro en varios países. El argumento central de estos autores es que las organizaciones sin fines de lucro están inmersas en instituciones y estructuras sociales y económicas. Esta teoría, considerando amplias relaciones de lo social, lo político y lo económico, identifica cuatro modelos de desarrollo institucional o tipo de régimen del tercer sector: a) el liberal, b) el social demócrata, c) el corporativista, y d) el estatista.

El Cuadro No 3 sugiere diversas hipótesis en cuanto a la relación entre el modelo institucional de desarrollo del sector sin fines de lucro y el voluntariado. Salamon y Sokolowski $(2001 ; 2003)$ sostienen que a medida que el voluntariado aumenta, el rol de estado disminuye y que la mayoría de los tipos de régimen o modelos institucionales enfatizan la orientación al servicio, con la excepción de los regímenes social demócratas donde las organizaciones orientadas a la expresión de necesidades son más comunes.
Salamon y Sokolowski (2001) sostienen que el voluntariado en América Latina presenta bajos niveles, al contextualizarlo en relación al tamaño de las economías de los países, y está orientado a las necesidades de servicio. El Cuadro $N^{\circ} 4$ presenta algunos hallazgos al utilizar la teoría de los orígenes sociales de la sociedad civil en América Latina. El tamaño del sector sin fines de lucro, el nivel de gasto social del gobierno, el rol de la religión y algunos antecedentes políticos y sociales fueron utilizados como criterios para clasificar a los países de acuerdo al patrón institucional. De acuerdo a lo postulado por la teoría, los datos confirman que en los países de patrón institucional estatista presentan un voluntariado orientado al servicio. Sin embargo, la orientación del voluntariado en México, país con patrón institucional estatista, no fue consistente con los postulados de la teoría que señalaban una orientación al servicio. El voluntariado en México, de acuerdo a los datos recogidos por estos autores, se caracteriza por tener orientación a la expresión de necesidades.

CUADRO NO 3

HIPÓTESIS DE LAS RELACIONES ENTRE LOS MODELOS INSTITUCIONALES DEL SECTOR SIN FINES DE LUCRO Y LA ESCALA Y TIPO DE VOLUNTARIADO

\begin{tabular}{|l|c|c|}
\hline Patrón Institucional & Voluntariado & $\begin{array}{c}\text { Tipo de Voluntariado } \\
\text { Dominante }\end{array}$ \\
\hline Social-demócrata & Alto & Expresión \\
\hline Liberal & Alto & Servicio \\
\hline Corporativista & Moderado & Servicio \\
\hline Estatista & Bajo & Servicio \\
\hline
\end{tabular}

Fuente: Salamon y Sokolowksi (2003, p. 81)

CUADRO NO 4

VOLUNTARIADO: PATRÓN INSTITUCIONAL, NIVEL, Y ORIENTACIÓN DOMINANTE POR PAÍS

\begin{tabular}{|l|l|l|l|}
\hline \multicolumn{1}{|c|}{ Country } & Patrón Institucional & Nivel (\%) & $\begin{array}{c}\text { Orientación Dominante } \\
\text { (Valores) }\end{array}$ \\
\hline Argentina & Corporativista & Moderado (2.5\%) & Servicio (70) \\
\hline Brasil & Estatista & Bajo* (0.3\%) & Servicio(94) \\
\hline Colombia & Estatista & Bajo (0.8\%) & Servicio (77) \\
\hline México & Estatista & Bajo (0.2\%) & Servicio (99) \\
\hline Perú & Estatista & Bajo (0.5\%) & Ambos 49) \\
\hline
\end{tabular}

*Bajo $=<1.5 \%$; Moderado $=>1.5 \%$ and $<3.5 \%$

Adaptado de Salamon y Sokolowski (2001) 
Esta teoría también describe el vínculo entre sociedad civil y voluntariado. Salamon y Sokolowski (2001; 2003) analizando datos provenientes de 24 países sugieren que tres fuerzas sociales han dado forma y desarrollo a las funciones del voluntariado: las relaciones de las clases sociales en el período de la industrialización, las políticas sociales gubernamentales y la religión organizada. Estos autores concluyen que el voluntariado puede ser entendido como un instrumento y un resultado de las políticas sociales que están finalmente conectadas con el patrón institucional de desarrollo o tipo de régimen de cada país.

Formalización. La formalización de las organizaciones ha sido uno de los focos de la sociología organizacional por más de cinco décadas. La investigación empírica en esta área ha sido guiada por el concepto de burocracia de Weber (Blauner, 1964; Pugh, et al., 1963). Hall (1962, 2002) es uno de los primeros autores que analiza empíricamente la formalización, en sus estudios sobre la dimensión burocrática de las organizaciones, utilizando los conceptos de "sistema de reglas" y "sistema de procedimientos". En general, los sociólogos organizacionales de la década del "60 entienden la formalización como un conjunto de reglas de la estructura organizativa (Blau \& Scott, 1962; Walsh $\&$ Dewar, 1987). Hall (1968) juntos a otros investigadores enfatizan en la formalización las reglas, procedimientos, comunicaciones e instrucciones escritas.

Uno de los primeros trabajos empíricos que analiza la formalización de las organizaciones de voluntariado fue desarrollado por Stuart Chapin y Tsouderos (1955; 1956) quienes analizan a través de métodos cuantitativos y cualitativos las organizaciones de voluntariado de St. Paul en Minnesota. Estos autores definen la formalización como el proceso a través del cual los grupos siguen patrones de procedimiento prescritos (Stuart Chapin \& Tsouderos, 1955). Estos autores señalan que la formalización de las organizaciones de voluntariado involucra un desarrollo secuencial de etapas caracterizado por: a) el aumento de la complejidad de la estructura social, b) una progresiva prescripción y estandarización de las relaciones sociales, y c) por un incremento de la burocratización de la organización (Stuart Chapin $\&$ Tsouderos, 1956)

En su primer estudio, Stuart Chapin y Tsouderos vinculan la formalización con las siguientes características de la estructura social de las organizaciones: a) criterio de membresía formal, b) funciones específicas para sus dirigentes, c) recambio de los directores y comités, d) recambio en los procedimientos de administración, e) incremento de la infraestructura, f) elaboración de políticas de personal, g) número de personas que reciben salario, y h) signos de conflicto dentro del sistema. De acuerdo a estos autores, el resultado final de la formalización corresponde a tres roles de los miembros de la organización: liderazgo, representación y personal administrativo.

Stuart Chapin y Tsouderos (1955) también identifican cinco etapas en el proceso de formalización: a) la etapa informal en la cual las organizaciones poseen características de los grupos primarios, tales como carencia de roles formales de autoridad y la autoridad basada en la personalidad del líder; b) la codificación de la estructura en la cual la persona y la posición son diferenciadas, emerge la jerarquía formal, se facilita la elección de las autoridades y surge la rotación de los roles formales; c) la diferenciación del ejecutivo en la cual surgen nuevas instancias tales como los comités y la dirección ejecutiva, se amplía la membresía y comienza el personal administrativo pagado; d) la multiplicación de las unidades de membresía en la cual varias unidades reproducen el proceso de formalización; y e) el control de la estructura en la cual se crean los comités especializados de control.

Stuart Chapin y Tsouderos (1956) señalan dos conclusiones principales de sus estudios de formalización del voluntariado: a) es un proceso uniforme $\mathrm{y}$ b) es un proceso que coincide con el crecimiento de la membresía. Algunas observaciones específicas de sus estudios incluyen: a) las organizaciones se formalizan independientemente de los objetivos principales de las mismas; b) las organizaciones extensas tienen solo un control relativo sobre la formalización de las organizaciones subsidiarias; c) la edad de la organización no es un factor central para explicar la formalización; y d) la velocidad del proceso de formalización varía enormemente.

Walsh y Dewar (1987) sostienen que el concepto de formalización ha sido estudiado principalmente desde una perspectiva empírica postergando su tratamiento teórico. Estos autores sugieren que la formalización puede ser entendida como un código, un canal y un estándar que promueve la eficiencia administrativa, sirve para el ejercicio del poder y las relaciones de autoridad en el contexto del desarrollo del ciclo de vida de la organización. La formalización, de acuerdo a estos autores, es un código en cuanto a que se dictan las reglas y los patrones de la conducta organizacional, un canal cuando conduce las relaciones humanas, y un estándar de la justicia 
distributiva cuando establece medidas para otorgar premios y castigos. Estos autores sostienen que estas tres funciones de la formalización contribuyen a la eficiencia y la influencia.

Walsh y Dewar (1987) identifican dos resultados de los procesos de formalización: la efectividad y eficiencia y la ineficacia y declive. Estos autores sostienen que en las primeras etapas del ciclo de desarrollo de las organizaciones, la formalización contribuye a la eficiencia. Sin embargo, en las etapas posteriores contribuye a su ineficiencia y declive. Por tanto, de acuerdo a estos autores los resultados de la formalización varían de acuerdo a las distintas etapas del ciclo de vida de las organizaciones.

La formalización ha sido asociada con diferentes características de las organizaciones, tales como tamaño (Mansfield, 1973), centralización, complejidad e innovación (Hall, 1968) y problemas de moral y enajenación, (Hage, 1965; Aiken \& Hage, 1966). También ha estado fuertemente asociado con reglas y procedimientos formales que se pueden sintetizar en el concepto de burocracia (Hall, 1962; Stuart Chapin y Tsouderos, 1955; 1956). Los investigadores de este tema también han vinculado la formalización con resultados contradictorios (eficiencia y declive) de acuerdo al desarrollo del ciclo de vida de la organización, y a procesos interrelacionados como el de profesionalización (Vollmer \& Mills, 1966).

El concepto de formalización casi desapareció de la reflexión académica debido en parte a la amplia y poco precisa utilización del concepto institucionalización. El concepto de institucionalización incluye a una diversidad de realidades y procesos empíricos (Scott, 2007). Este estudio hace una opción por el concepto de formalización debido a su mayor precisión en cuanto a la realidad empírica que pretende capturar: tipos y características de los programas y la participación de los voluntarios.

\section{Modelo conceptual e hipótesis de investigación}

El argumento central de la teoría de los orígenes sociales de la sociedad civil es que hay factores estructurales que aumentan o reducen el tamaño, composición y orientación del sector sin fines de lucro o sociedad civil. De este modo, las organizaciones y programas de voluntariado juvenil pueden crecer o disminuir de acuerdo a la influencia de macro-variables. En el modelo planteado, las variables a nivel macro consideradas son: el nivel de inequidad (Coeficiente de Gini), el nivel de pobreza y el ingreso nacional.
Para entender el voluntariado desde una perspectiva más comprehensiva es necesario situarlo en el contexto de instituciones y fuerzas sociales amplias. En este estudio el voluntariado es entendido en el contexto de la sociedad civil. De este modo, las concepciones de construcción de ciudadanía, profundización de la democracia y disminución de la pobreza están lógicamente asociadas al voluntariado. También el rol de la cultura y la religión son importantes para situar y comprender el voluntariado. Si bien existen factores individuales y psicológicos asociados al voluntariado (Wilson, 2000) en el cual los valores, creencias y motivaciones son importantes, este estudio se concentra en variables macro y estructurales a nivel de país, organización y programa.

Al considerar las teorías y estudios previos, los vacíos en la investigación empírica en esta área son múltiples, sin embargo, al menos tres nuevos aspectos son abordados en este estudio: a) algunos aspectos de la teoría de los orígenes sociales de la sociedad civil serán sometidos a prueba considerando datos de algunos países de América del sur, b) este estudio incluye variables a nivel nacional para entender variaciones del voluntariado juvenil en el contexto de América del sur y c) el foco de estudio son los jóvenes, los cuales constituyen junto a los niños el segmento de la población mayoritario de la región.

El modelo propuesto está fortalecido por la incorporación de variables que han sido parcialmente consideradas por la teoría de los orígenes sociales de la sociedad civil: el sector público y la economía. Las variables del sector público incluidas en el modelo son: el presupuesto nacional, la efectividad del gobierno y el nivel de gasto social. Además, el modelo integra tres aspectos cruciales asociados a los problemas centrales de la región: el nivel de pobreza, el nivel de inequidad (Coeficiente de Gini) y el ingreso nacional. El modelo analítico está representado en la Figura № 2 , en la cual se observan tres niveles: programa, organización y país (nacional). Las variables dependientes de este modelo son la orientación al servicio y el nivel de formalización.

A partir de este esquema, se postulan los siguientes planteamientos teóricos: a) los tres niveles analíticos son importantes para explicar variaciones en las variables dependientes; b) en nivel nacional, las características de la economía y el sector público influencian las variables dependientes; c) las variables organizacionales orientación religiosa, orientación educacional y nivel de operación influencian las variables dependientes; d) Las variables a nivel de 
programa liderazgo juvenil, compromiso de tiempo y programa incluyente influencian el nivel de formalización y la orientación al servicio. Considerando el número de variables, los niveles incluidos en el modelo y el carácter exploratorio de esta indagación, las relaciones e hipótesis posibles de plantear son múltiples, sin embargo se especifican sólo algunas.

a. Se espera que el ingreso nacional tenga un efecto positivo en la formalización de los programas de voluntariado y negativo con respecto a la orientación al servicio. Mientras mayor ingreso tenga un país, mayores serán los recursos disponibles para las organizaciones de voluntariado, por tanto, se observarán mayores niveles de formalización del voluntariado. Además, las organizaciones y programas de voluntariado estarán más proclives a orientar sus acciones en torno a la satisfacción de las necesidades de expresión, no así a las de servicio.

b. El nivel de pobreza e inequidad tendrán una influencia negativa en cuanto al nivel de formalización de las organizaciones y programas de voluntariado, por tanto éstos serán menos formales, y positiva en cuanto a la orientación a las actividades de servicio.

c. Se espera que la efectividad del gobierno tenga un efecto negativo en el nivel de formalización de los programas de voluntariado. Esta relación está basada en la idea de que las organizaciones de la sociedad civil complementan la acción del estado ("cubren la brecha") o estarían desarrollando actividades en las áreas que el estado, por ausencia de recursos u otras razonas, ha descuidado.

d. En el nivel organizacional, se espera que la orientación religiosa, la orientación educacional y el nivel de operación influyan positivamente en el nivel de formalización de los programas. Una explicación parcial de esta relación estaría dada por la influencia que tienen las altamente estructuradas y jerarquizadas organizaciones religiosas y educacionales en sus programas. Respecto al nivel de operación, se espera que las organizaciones y programas que desarrollan sus actividades en distintos niveles (local, regional, nacional) tendrán un mayor nivel de formalización.

e. En el nivel de programa, se espera que el liderazgo juvenil y el compromiso de tiempo tengan una relación positiva con el nivel de formalización. La mayor participación y nivel de involucramiento de los jóvenes redundará en un mayor nivel de formalización de los programas. Los programas incluyentes estarían negativamente asociados con la formalización del voluntariado debido a que los programas con menores requisitos de elegibilidad (más inclusivos) son menos formales que los programas con mayor número de requisitos para que los jóvenes participen en ellos (más excluyentes).

Las hipótesis a nivel de organización y programa están parcialmente basadas en dos estudios de casos realizados por el autor en dos organizaciones de voluntariado: "Un techo para Chile" y "Opción Colombia" (Olate, et al., 2006). Las posibilidades de identificar y profundizar en todas las potenciales relaciones del modelo planteado quedan como una tarea pendiente.

Este estudio busca responder específicamente a la pregunta: ¿Cuáles son las variables que influyen en la orientación y formalización de los programas de voluntariado juvenil en el nivel nacional, de organización y de programa? Las hipótesis del estudio están planteadas por nivel:

A nivel nacional:

1. La efectividad del gobierno está negativamente asociada con el nivel de formalización.

A nivel organizacional:

2. El nivel de operación está positivamente asociado con el nivel de formalización.

A nivel de programa:

3. El liderazgo juvenil está positivamente asociado con el nivel de formalización.

4. El compromiso de tiempo está positivamente asociado con el nivel de formalización.

5. Los programas incluyentes están positivamente asociados con el nivel de formalización. 


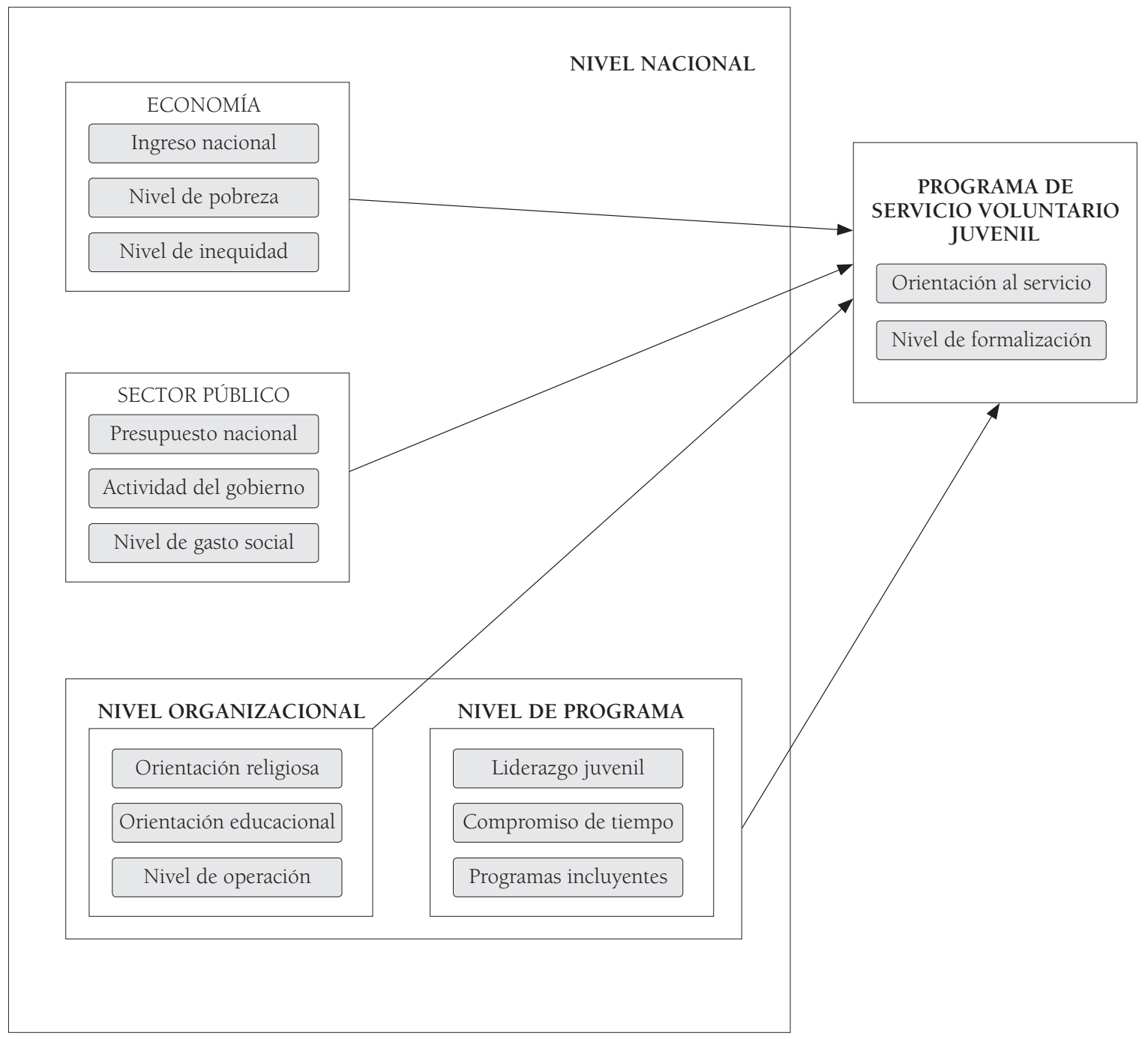

Metodología y muestra. Este estudio utiliza la base de datos del estudio desarrollado por el Centro para el Desarrollo Social de Washington University titulado "Servicio Voluntario Juvenil en América Latina y el Caribe: Un estudio regional (2006-2007)". El universo del estudio del Centro para el Desarrollo Social de Washington University estaba constituido por los 26 países miembros del Banco Interamericano de Desarrollo (BID). Debido a diversas restricciones, se utilizó un método cuasi-probabilístico y de múltiples etapas para la construcción de la muestra. La región de América Latina y el Caribe fue estratificada siguiendo dos criterios: geográfico y población de los países. De este modo, se establecieron las subregiones de el Caribe (Guyana Jamaica y República Dominicana), América Central y México (Guatemala, Nicaragua, México y Panamá), y América del Sur (Ver Cuadro No5). En América del Sur se seleccionó a Brasil por ser el país con mayor población, aproximadamente 195 millones de personas (CEPAL, 2007). Utilizando la técnica de muestro probabilístico proporcional al tamaño, cuatro países adicionales fueron seleccionados en la subregión de América del Sur. Por tanto, la base de datos incluye 304 programas pertenecientes a 223 organizaciones en 5 países. El porcentaje de retorno de las encuestas para América del sur corresponde al 71.93\% (223/310). Para América Latina, la base de datos incluye 533 programas pertenecientes a 374 organizaciones en 12 países. 
CUADRO N ${ }^{\circ} 5$

MUESTRA: ORGANIZACIONES Y PROGRAMAS

\begin{tabular}{|l|c|c|c|c|}
\hline & $\begin{array}{c}\text { Número de } \\
\text { Organizaciones } \\
\text { Identificadas }\end{array}$ & $\begin{array}{c}\text { Número de } \\
\text { Organizaciones } \\
\text { Encuestadas }\end{array}$ & $\begin{array}{c}\text { Número de } \\
\text { Programas } \\
\text { Encuestados }\end{array}$ & $\begin{array}{c}\text { Porcentaje de } \\
\text { Retorno de } \\
\text { Encuestas }\end{array}$ \\
\hline Argentina - Bs. Aires & 63 & 32 & 55 & $50.79 \%$ \\
\hline Brasil - Sao Paulo & 101 & 65 & 104 & $64.36 \%$ \\
\hline Colombia - Bogotá & 24 & 21 & 26 & $87.50 \%$ \\
\hline Perú - Lima & 101 & 94 & 105 & $93.07 \%$ \\
\hline Venezuela - Caracas & 21 & 11 & 14 & $52.38 \%$ \\
\hline América del Sur & 310 & 223 & 304 & $71.93 \%$ \\
\hline América Latina y el Caribe & 516 & 374 & 533 & $72.48 \%$ \\
\hline
\end{tabular}

El método de censo fue utilizado para seleccionar los programas de voluntariado juvenil en la ciudad con mayor población de cada país. La unidad de análisis de esta investigación son los programas de voluntariado definidos como un sistema coordinado formal de actividades con distintos objetivos (McBride, Olate, \& Johnson, 2008). Para que los programas de voluntariado fuesen incluidos en la muestra debían cumplir con los siguientes requisitos: a) apoyado por una organización, b) tener un nombre, c) tener un presupuesto, d) tener un rol definido para los voluntarios, y e) que la mayoría de sus voluntarios fuesen jóvenes entre 15 y 29 años.

Cuestionario. El cuestionario fue desarrollado por el equipo de investigación del proyecto Servicio Voluntario Juvenil que incluye a la Fundación SES de Argentina, el Programa de Alcance Juvenil del BID (BID Juventud) y el Centro para Desarrollo Social (CDS) de Washington University. El cuestionario contiene preguntas en dos niveles (organización y programa) y fue respondido por los responsables de programas entre septiembre de 2006 y junio de 2007. El cuestionario estuvo disponible en tres idiomas: ingles, español y portugués, e incluyó 48 preguntas relativas al programa (actividades y objetivos, rol del voluntario, características del personal administrativo y de los voluntarios, identidad y administración) y 17 preguntas relativas a la organización (identificación, historia, tipo, nivel de operación, personal administrativo y voluntarios, y financiamiento) donde el programa se desarrolla. El cuestionario fue desarrollado primero en inglés, luego de la primera prueba fue traducido al español y después de la segunda prueba fue traducido al portugués. Se implementaron cuatro pruebas del cuestionario, entre noviembre del 2005 y agosto del 2006, para verificar los procedimientos de recolección de datos y el cuestionario.

Estas pruebas evaluaban tres dimensiones: las preguntas específicas (variación, significado, nivel de dificultad e interés de los consultados), el cuestionario como un todo (secuencia de las secciones y orden de las preguntas) (Converse \& Presser, 1986), y procedimientos para recolectar los cuestionarios (comunicación entre los investigadores de campo y Fundación SES, BID Juventud y el equipo del CDS). Diferentes versiones del cuestionario fueron probadas entre una y tres veces en 22 organizaciones de voluntariado juvenil de la región. Las organizaciones en las cuales fue probado el cuestionario están localizadas en países o ciudades que no fueron parte de la muestra. Además, varios expertos, académicos, y administradores de programas de voluntariado juvenil fueron consultados acerca del contenido y el formato del cuestionario y los procedimientos de recolección de datos. Las encuestas fueron enviadas como documentos adjuntos por correo electrónico en formato de formulario del programa Microsoft Word a cada administrador o líder de la organización junto a una carta de invitación.

Variables. Las Variables dependientes. La primera variable dependiente, orientación al servicio, fue construida a partir de una adaptación de la Clasificación Internacional de las Organizaciones sin fines de lucro (ICNPO). Las organizaciones participantes en el estudio fueron inicialmente clasificadas en 14 categorías, para luego ser reagrupadas en dos categorías: orientación al servicio y orientación a la expresión.

La segunda variable dependiente, nivel de forma- 
lización, fue construida a partir de la aplicación de dos técnicas estadísticas: coeficiente Alfa de Cronbach y análisis de factores principales. El coeficiente alfa fue utilizado para determinar el grado de correlación entre las 36 variables iníciales del índice, y posteriormente se utilizó el análisis de factores principales El objetivo de utilizar ambas técnicas fue la búsqueda de unidimensionalidad y consistencia interna. Las variables que incluye el índice de formalización son: 1) Apoyo: Talleres de reflexión; 2) Compensación: Ropas; 3) Reconocimiento: vestimentas y/o libros y mochilas; 4) Reconocimiento: participación en conferencias; 5) Identidad: Gorros; 6) Identidad: Artículos de escritorio; 7) Identidad: Bolsos. El Índice de Formalización tiene siete ítems y un $\alpha=.7077$, lo cual indica una medida aceptable de la formalización de los programas de voluntariado (Ver Cuadro No 6).

Se puede observar que tres de las siete variables corresponden a la dimensión aspectos de la identidad del programa, dos corresponden a la dimensión de reconocimiento, una variable a la dimensión de apoyo al rol del voluntario, y una variable a la dimensión de compensación. De las siete variables del índice, cinco están vinculadas a aspectos de marketing social: gorras, artículos de escritorio, bolsos, ropas, libros y mochilas. Por tanto, este índice sugiere que los programas de voluntariado juvenil tienen una formalización empírica asociada a estrategias de marketing social.

\section{CUADRO NO 6 \\ ÍNDICE DE FORMALIZACIÓN POR PAÍS Y SUBREGIÓN}

\begin{tabular}{|l|c|}
\hline País & Índice de Formalización \\
\hline Brasil & 2.538 \\
Colombia & 1.962 \\
Perú & 1.886 \\
Argentina & 1.673 \\
Venezuela & 1.357 \\
\hline América del Sur & 2.053 \\
\hline América Latina y el Caribe & 1.893 \\
\hline
\end{tabular}

Variables independientes en el nivel de programa y organización. Las variables en el nivel de organización incluyen: orientación religiosa ( $\mathrm{Si} / \mathrm{No}$ ), orientación educacional (Si/No) y nivel de operación (rango de 1 a 6). En el nivel de programa las variables incluyen: liderazgo juvenil (rango de 1 a 6), compromiso de tiempo (rango de 1 a 4), y programas incluyentes (rango de 1 a 12). Las variables del nivel de programa y organización fueron obtenidas de la base de datos del estudio del Centro para el Desarrollo Social de Washington University.

Variables independientes en el nivel nacional. Las variables en el nivel nacional fueron organizadas en dos categorías: sector público y economía. Se utilizaron tres variables proxi para la medición del sector público: ingreso nacional, efectividad del gobierno y nivel del gasto social. La economía nacional fue medida utilizando tres variables: ingreso nacional, el nivel de pobreza, y el nivel de equidad (coeficiente Gini). Las fuentes de los datos del nivel nacional corresponden al Banco Mundial (World Bank, 2007) y la Comisión Económica para América Latina (ECLAC, 2007).

Análisis de datos. El análisis de los datos involucró un plan de cuatro etapas: a) preparación y limpieza de los datos; b) test de asociación entre variables; c) desarrollo del path análisis; y d) análisis de las hipótesis y preguntas de investigación. En la primera etapa se examinaron distribuciones de frecuencia y datos perdidos. Los datos perdidos de las variables en el nivel organizacional y de programa oscilaron entre menos de un $1 \%$ y $4.6 \%$. Previo a los procedimientos de regresión se realizaron diagnósticos para comprobar que las premisas de la regresión fuesen cumplidas y detectar problemas de multicolinealidad. La segunda etapa buscaba diagnosticar las correlaciones existentes entre la segunda variable dependiente, orientación al servicio, y las variables independientes, para ello se utilizó el t-test. Todas estas técnicas estadísticas fueron ejecutadas utilizando el paquete computacional Stata 10.0.

En la tercera parte se realizó el trabajo de preparación y desarrollo del path análisis. Se utilizó inicialmente el análisis multivariable de varianza (MANOVA), Posteriormente se ejecutó el path análisis para lo cual se utilizó el programa estadístico AMOS 16. Para el desarrollo del path análisis se siguieron dos pasos: calce inicial del modelo y depuración del modelo. Para el calce inicial del modelo se utilizó MANOVA, descartando aquellas variables que no estuvieran significativamente asociadas con la variable dependiente. La depuración del modelo consistió en la eliminación de aquellos parámetros no significativos del path análisis (Schumaker \& Lomax, 2004). Finalmente, se procedió a responder las preguntas de investigación y a analizar las hipótesis. 


\section{Resultados}

Asociaciones con la primera variable dependiente: Orientación al Servicio. Los resultados del análisis de 12 t-tests independientes que buscaban establecer la correlación entre las variables independientes y la Orientación al Servicio no encontraron ninguna diferencia estadísticamente significativa entre las variables. Debido al alto número de t-test ejecutados, es muy posible que este importante hallazgo corresponda a un error Tipo I. Como resultado de estos análisis, la variable orientación al servicio es removida del modelo, por tanto, el Índice de Formalización queda como única variable dependiente en el modelo.

Asociaciones con el Índice de Formalización. Las variables encontradas con una asociación estadísticamente significativas a un valor de $p=.05$ con el análisis multivariable de varianza (MANOVA), fueron incluidas en el path análisis. Las MANOVAs fueron ejecutadas en cuatro regresiones, una para los niveles de programa y organización y dos para el nivel nacional (Ver Cuadro $N^{\circ}$ 7). Se encontraron cuatro variables asociadas de manera estadísticamente significativa con la variable dependiente: Liderazgo Juvenil y Programas Incluyentes en el nivel de programa, y Nivel de Pobreza y Efectividad del Gobierno en el nivel nacional. No se encontraron relaciones significativas a nivel de organizacional.

En la Figura 3 se observan en el path análisis las variables significativamente asociadas al índice de formalización para la muestra de programas de América Latina y el Caribe ( $\mathrm{n}=533$ ). El RMSEA (root mean square error of approximation), para este modelo es 0 , con un $90 \%$ de intervalo de confianza que va de un 0 a un .064 , lo cual sugiere un buen calce del modelo a los datos. Este enunciado está apoyado por un Chi-cuadrado $\mathrm{X}^{2}(\mathrm{p}=.543)$ estadísticamente no significativo de 2.145 con 3 grados de libertad, lo cual indica que el modelo es aceptable. Los resultados indican que este modelo explica el 13\% de la varianza del índice de formalización.

CUADRO NO 7

MANOVAS POR NIVEL

\begin{tabular}{|c|c|c|c|c|c|}
\hline & Wilks' Lambda & $\mathrm{eta}^{2}$ & df & F & Prob $>F$ \\
\hline \multicolumn{6}{|l|}{ Nivel de Programa: } \\
\hline Índice de Formalización & .8890 & .1110 & 3 & 21.35 & $.0000 *$ \\
\hline Liderazgo Juvenil & .9236 & .0764 & 1 & 42.45 & $.0000 *$ \\
\hline Compromiso de Tiempo & .9993 & .0007 & 1 & .33 & .5631 \\
\hline Programas Incluyentes & .9625 & .0375 & 1 & 19.97 & $.0000^{*}$ \\
\hline \multicolumn{6}{|l|}{ Nivel de Organización: } \\
\hline Índice de Formalización & .9984 & .0016 & 3 & .28 & .8373 \\
\hline Orientación Religiosa & .9996 & .0004 & 1 & .22 & .6359 \\
\hline Orientación Educacional & .9998 & .0002 & 1 & .08 & .7733 \\
\hline Nivel de Operación & 9989 & .0011 & 1 & .58 & .4448 \\
\hline \multicolumn{6}{|l|}{ Nivel Nacional: } \\
\hline Índice de Formalización & .9610 & .0390 & 3 & 6.16 & $.0004^{*}$ \\
\hline Presupuesto Nacional & .9922 & .0078 & 1 & 3.57 & .0594 \\
\hline Efectividad del Gobierno & .9800 & .0200 & 1 & 9.33 & $.0024 *$ \\
\hline Gasto Social & .9919 & .0081 & 1 & 3.72 & .0544 \\
\hline Índice de Formalización & .9555 & .0445 & 3 & 8.21 & $.0000 *$ \\
\hline Ingreso Nacional & .9946 & .0054 & 1 & 2.89 & .0895 \\
\hline Nivel de Pobreza & .9682 & .0318 & 1 & 17.38 & $.0000 *$ \\
\hline Nivel de Inequidad & .9954 & .0046 & 1 & 2.44 & .1186 \\
\hline
\end{tabular}




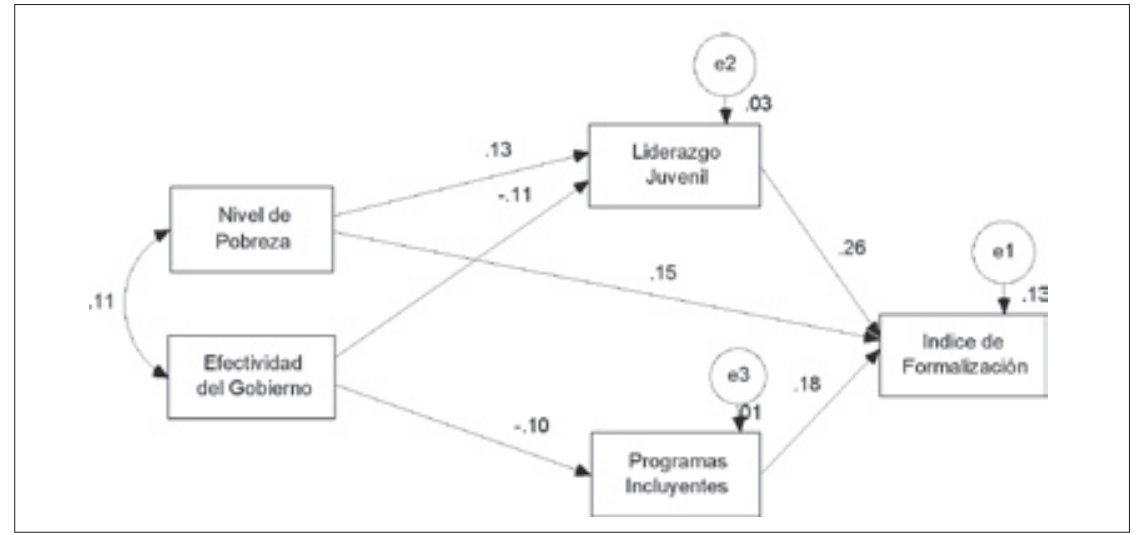

En la Figura No 4 se observan en el path análisis algunas variables significativamente asociadas al índice de formalización para la muestra de programas de América del Sur $(\mathrm{n}=310)$. El RMSEA (root mean square error of approximation), para este modelo es .068 , con un $90 \%$ de intervalo de confianza que va de un 0 a un .134, lo cual sugiere que el modelo no se ajusta a los datos. Este enunciado es confirmado por Chi-cuadrado $\mathrm{X}^{2}(\mathrm{p}=.064)$ estadísticamente significativo de 7.257 con 3 grados de libertad, lo cual indica que el modelo no es aceptable (Ver Cuadro No 8).

\section{FIGURA N ${ }^{\circ} 4$}

FACTORES QUE INFLUENCIAN LA FORMALIZACIÓN DEL VOLUNTARIADO EN AMÉRICA DEL SUR

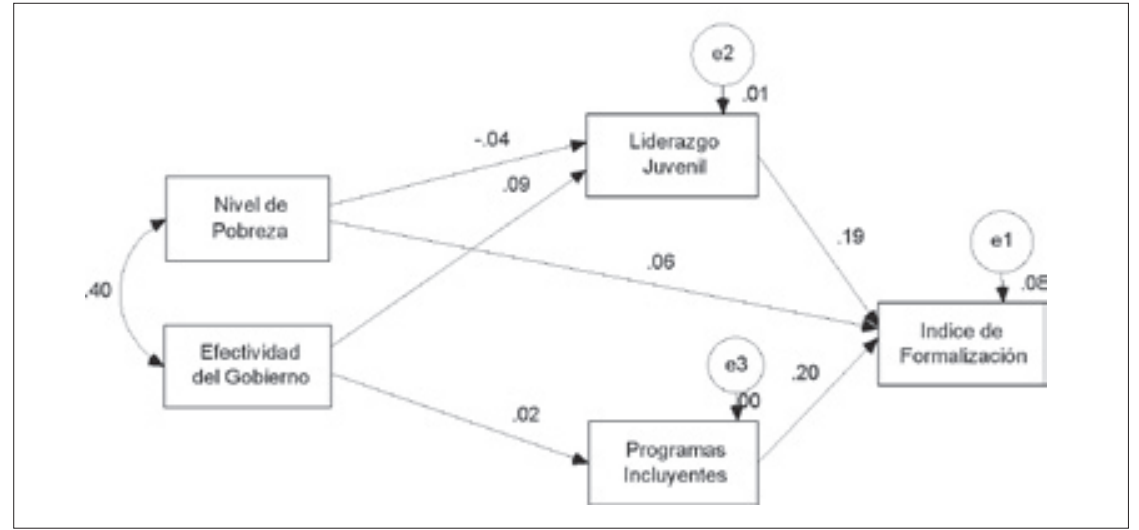

CUADRO No 8

COMPARACIÓN DE LOS CRITERIOS DE AJUSTE DE LOS MODELOS DE ANÁLISIS DE SENDEROS

\begin{tabular}{|l|c|c|}
\hline & América Latina & América del Sur \\
\hline $\mathrm{N}$ & 533 & 304 \\
\hline $\mathrm{X}^{2}$ & 2.145 & 7.257 \\
\hline $\mathrm{Df}$ & 3 & 3 \\
\hline $\mathrm{P}$ & .543 & .064 \\
\hline $\mathrm{r}^{2}$ & .129 & .08 \\
\hline RMSEA & .000 & .068 \\
\hline LO 90 & .000 & .000 \\
\hline HI 90 & .064 & .134 \\
\hline
\end{tabular}


El modelo No3 que utiliza los datos de América Latina y el Caribe, es un modelo resuelto y estandarizado que tiene siete parámetros (seis senderos-paths- y la correlación entre las variables a nivel nacional). A continuación se describen las relaciones entre las variables, comenzando por las variables en el nivel nacional (de izquierda a derecha). La percepción de la Efectividad del Gobierno tiene un efecto moderado y negativo (.-10, p=.019) en que los Programas sean Incluyentes y un moderado efecto negativo (.11, p=.010) en el Liderazgo Juvenil. El Nivel de Pobreza tiene un moderado efecto positivo (.13, $\mathrm{p}=.003$ ) en el Liderazgo Juvenil y también un moderado efecto positivo $(.15, \mathrm{p}<.001)$ en el Índice de Formalización. El Liderazgo Juvenil tiene a su vez un fuerte efecto positivo (.26, p<.001) en el Índice de Formalización. Los Programas Incluyentes tienen un moderado efecto positivo $(.18, \mathrm{p}<.001)$ en el Índice de Formalización.

El modelo No 4 que utiliza los datos de América del Sur, es un modelo no resuelto y no estandarizado que tiene sólo 2 asociaciones estadísticamente significativas: el Liderazgo Juvenil tiene un moderado efecto positivo $(.19, \mathrm{p}<.001)$ en el Índice de Formalización y los Programas Incluyentes tienen un moderado efecto positivo (.20, p<.001) en el Índice de Formalización. En conclusión, sólo el modelo que utiliza los datos para América Latina y el Caribe es aceptado como una buena explicación de las relaciones entre el índice de formalización y las variables en el nivel de programa (liderazgo juvenil y programas incluyentes) y en el nivel nacional (nivel de pobreza y efectividad del gobierno). El modelo que utiliza los datos de América del Sur identifica relaciones significativas con el Índice de Formalización sólo en el nivel de programa: Liderazgo Juvenil y Programas Incluyentes.

De las cinco hipótesis planteadas, las hipótesis 1 , 2 y 4 son rechazadas. Respecto a la hipótesis uno, la Efectividad del Gobierno tiene una relación indirecta con el Índice de Formalización que pasa a través de la relación estadísticamente significativa y negativa de Programas Incluyentes, para los datos de América Latina y el Caribe. Las hipótesis 3 y 5 , postuladas en el nivel de programa, encontraron evidencia positiva: a mayor liderazgo juvenil mayores niveles de formalización y los programas más incluyentes son más formales. Por tanto, son aceptadas las hipótesis 3 y 5 tanto para el modelo de América Latina y el Caribe como para el modelo de América del Sur.

Respecto al resto de las hipótesis: Hipótesis 2: no se encontró evidencia de relaciones estadísticamente significativas en las variables del nivel organizacional, el Nivel de Operación de la organización no esta relacionado con el Índice de Formalización. Hipótesis 4: no existe una relación estadísticamente significativa entre el Compromiso de Tiempo de los jóvenes para realizar sus actividades voluntarias y el Índice de Formalización.

\section{Conclusión}

Este estudio constituye un aporte a la investigación empírica respecto a la orientación y formalización del voluntariado en América del sur utilizando un modelo conceptual de tres niveles basado en la teoría de los orígenes sociales de la sociedad civil. El modelo analítico fue desarrollado para entender empíricamente los factores asociados a la orientación y formalización de los programas de voluntariado juvenil. Estos factores son: la efectividad del gobierno y el nivel de pobreza, en el nivel nacional, y el liderazgo juvenil y los programas incluyentes, en el nivel de programa. Estas relaciones encontraron evidencia utilizando los datos para América Latina y el Caribe. Para los datos de América del Sur, sólo el liderazgo juvenil y los programas incluyentes están asociados al nivel de formalización.

La intuición básica de la teoría de los orígenes sociales de la sociedad civil pudo ser comprobada en este análisis empírico, existen variables a nivel nacional (efectividad del gobierno y nivel de pobreza) que pueden explicar algunas características del voluntariado (formalización). Sin embargo, el segundo aspecto de esta teoría que fue analizado, la orientación del voluntariado, vinculado a los patrones institucionales de desarrollo del tercer sector no encontró sustento en esta base de datos de programas y organizaciones de voluntariado juvenil.

Los resultados de esta investigación deben ser considerados con precaución debido a las limitaciones metodológicas, de recolección de datos, teóricas y de diseño del estudio. Estas limitaciones incluyen: la utilización de varios idiomas en los cuestionarios; la utilización de diferentes técnicas para la recolección de datos (email, teléfono y entrevista); la imposibilidad de que los investigadores de campo accedieran a todas las organizaciones de voluntariado de cada ciudad; el carácter exploratorio de la investigación; las dificultades para acceder a datos confiables en el nivel nacional que permitan la utilización de un diseño multi-niveles; y las obvias limitaciones para establecer causalidad por tratarse de datos transversales.

La asociación entre la formalización de los programas de voluntariado juvenil y el impacto que 
estos programas tienen en las comunidades y en los mismos voluntarios se considera un elemento fundamental. Este estudio intenta destacar, a través del desarrollo de una medición estadística y empírica, la importancia de la formalización y orientación de estos programas. Una hipótesis para futuros estudios queda planteada: los programas de voluntariado juvenil con mayores niveles de formalización tienen a través de sus actividades mayores niveles de impacto social, económico y político. Finalmente, considerando el alto porcentaje de jóvenes en la región y el potencial crecimiento de los programas de voluntariado juvenil, es necesario realizar más estudios específicos, especialmente investigaciones y propuestas de programas que incorporen a los jóvenes marginados no sólo como beneficiarios sino como actores del voluntariado.

\section{Referencias}

AIKEN, M. \& HAGE, J. (1968). Organizational interdependence and intraorganizational structure. American Sociological Review, 33, 912-930.

BLAU, P.M. \& SCOTT, W.R. (1962). Formal organizations: A comparative approach. San Francisco, CA: Chandler.

BLAUNER, R. (1964). Alienation and freedom. Chicago: University of Chicago Press.

CIVICUS, IAVE, \& UNV. (2008). Volunteering and social activism. Pathways for participation in human development. El documento fue obtenido en 30 de octubre de 2008, desde: http://www.unv.org/fileadmin/img/wvw/ Volunteering_and_Social_Activism_FINAL.pdf

CONVERSE, J. \& PRESSER, S. (1986). Survey questions. Handcrafting the questionnaire. Thousand Oak, California: Sage Publications.

ECONOMIC COMMISSION FOR LATIN AMERICA AND THE CARIBBEAN, ECLAC (2007). Statistical yearbook for the Latin American and the Caribbean. Santiago, Chile: United Nations.

HAGE, J. (1965). An axiomatic theory of organizations. Administrative Science Quarterly, 10, 289-320

HALL, R. H. (1962). The concept of bureaucracy: an empirical assessment. American Journal of Sociology, 69, 32-40.

HALL, R. H. (1968). Professionals and bureaucratization. American Sociological Review, 33, 90-103.

HALL, R. H. (2002). Organizations: Structures, Processes, and Outcomes. (8th ed.). Prentice-Hall, London.

HASENFELD, Y. \& GIDRON, B. (2005). Understanding multi-purpose hybrid voluntary organizations: The contributions of theories on civil society, social movements and non-profit organizations. Journal of Civil Society, 1(2), 97 - 112.
INGLEHART, R. (2003). Modernization and volunteering. In P. Dekker \& L. Halman (Eds.), The values of volunteering. Cross-cultural perspectives. New York: Kluwer Academic / Plenum Publishers.

KAUFMANN, D., KRAAY, A. \& MASTRUZZI, M. (2007). Governance matters VI: Aggregate and individual governance indicators 1996-2006. Policy Research Working Paper 4280. Washington, DC: World Bank.

MANSFIELD, R. (1973). Bureaucracy and centralization: an examination of organizational structure. Administrative Science Quarterly, 18, 477-488.

MCBRIDE, A.M., OLATE, R. \& JOHNSON, L. (2008). Youth volunteer service in Latin America and the Caribbean: A regional assessment (CSD Research Report RP 08-05). St. Louis: Center for Social Development, Washington University.

O'CONNOR, T. R. \& ESKEY, M.T. (2005). Types of scales and indexes. In Kempf-Leonard, K. (Ed.), Encyclopedia of social measurement (pp. 443-453. Vol. 3). Oxford: Elsevier Academic Press.

OLATE, R. (2007). Youth volunteerism in Latin America and the Caribbean: the formalization of voluntary service and the theory of social origins. Tesis de doctorado. Washington University.

OLATE, R., JOHNSON, L. \& MCBRIDE A.M. (2007). Servicio voluntario juvenil en América Latina y el Caribe: historia, tendencias y potencialidades. In Solari, A., \& Cruz, A. (Ed.) Sociedad civil y desarrollo local (pp. 165-183), México, DF: Porrúa.

OLATE, R., MCBRIDE, A. \& JOHNSON, L. (2006). From new social movements to civic service organizations: The case of "Opción Colombia" and "Un Techo para Chile." Unpublished manuscript. George Warren Brown School of Social Work, Washington University, St. Louis.

PUGH, D.S., HICKSON, D.J., HININGS, C.R., MACDONALD, K.M., TURNER, C. \& LUPTON, T. (1963). A conceptual scheme for organizational analysis. Administrative Science Quarterly, 8, 289-315.

PUGH, D.S., HICKSON, D.J., HININGS, C.R. \& TURNER, C. (1963). Dimensions of organizational structure. Administrative Science Quarterly, 13, 65-105.

SALAMON, L. \& ANHEIER, H. (1992). In search of the nonprofit sector II: The problem of classification. Baltimore: The Johns Hopkins Institute for Policy Studies. Working Paper No.3.

SALAMON, L. \& ANHEIER, H. (1996). The international classification of nonprofit organizations: ICNPO-Revision 1, 1996. Baltimore: The Johns Hopkins Institute for Policy Studies. Working Paper No.19.

SALAMON, L. \& ANHEIER, H. (1998). Social origins of civil society: Explaining the nonprofit sector cross-nationally. Voluntas: International Journal of Voluntary and Nonprofit Organizations, 9(3), 213-248. 
SALAMON, L., SOKOLOWSKI, W. \& ANHEIER, H. (2000). Social origins of civil society: An overview (Working Paper No. 38). Baltimore: The Johns Hopkins Center for Civil Society Studies.

SALAMON, L. \& SOKOLOWSKI, W. (2001). Volunteering in cross-national perspective: Evidence from 24 countries (Working paper No. 40). Baltimore: Center for Civil Society Studies, Johns Hopkins University.

SALAMON, L. \& SOKOLOWSKI, W. (2003). Institutional roots of volunteering. Toward a macro-structural theory of individual voluntary action. In P. Dekker \& L. Halman (Eds.), The values of volunteering. Cross-cultural perspectives (pp. 71-90). New York: Kluwer Academic / Plenum Publishers.

SALAMON, L. \& SOKOLOWSKI, W. (2004). Global civil society. Dimensions of the nonprofit sector, volume two. Bloomfield, CT: Kumarian Press.

SCHUMAKER, R. E., \& LOMAX (2004). A beginner's guide to structural equation modeling (2nd ed.). New Jersey: Lawrence Erlbaum Associates.

SCOTT, W.R. (2007). Institutions and Organizations: Ideas and Interests (3rd ed.). Thousand Oak, California: Sage Publications.

STUART CHAPIN, F. \& TSOUDEROS, J.E. (1955). Formalization observed in ten voluntary associations: Concepts, morphology, process. Social Forces, 33 (4), 306-309.

STUART CHAPIN, F. \& TSOUDEROS, J.E. (1956). Formalization observed in ten voluntary associations: Concepts, morphology, process. Social Forces, 34, (4), 342-344.

UNITED NATIONS (2008a). Report of the Secretary-General. Follow-up to the implementation of the International Year of Volunteers. New York: United Nations. El documento fue obtenido el 30 de octubre de 2008, desde http://www.worldvolunteerweb.org/fileadmin/docdb/ pdf/2008/World_Volunteer_Web_stuff/UNRes_A_63_ 184.pdf

UNITED NATIONS (2008b). The Millenium Development Goals Report. New York: United Nations. El documento fue obtenido el 10 de noviembre de 2008, desde http:// www.un.org/millenniumgoals/pdf/The\%20Millennium \%20Development\%20Goals\%20Report\%202008.pdf

UNITED NATIONS (2005a). Report of the Secretary-General. Follow-up to the implementation of the International Year of Volunteers. New York: United Nations. Retrieved July 10, 2006 from http://www.worldvolunteerweb. org/fileadmin/docs/old/pdf/2005/050830INT_res_ iyvffup.pdf

UNITED NATIONS (2005b). The Millennium Development Goals Report 2005. New York: United Nations.
UNITED NATIONS VOLUNTEERS UNV (2001). Expert working group meeting on volunteering and social development. New York, 29-30 November 1999. Bonn: United Nations Volunteers.

VELTMEYER, H. (2004). Civil society and social movements. The dynamic of intersectoral alliances and urban-rural linkages in Latin America. (Program Paper 10). New York: United Nations Research Institute for Social Development.

VOLMER, H. M. \& MILLS, D. L. (1966). Professionalization. Englewood Cliffs, NJ: Prentice-Hall.

WALSH, J.P. \& DEWAR, R.D. (1987). Formalization and the organization life cycle. Journal of Management Studies, 24(3), 215-231.

WILSON, J. (2000). Volunteering. Annual Review of Sociology, 26, 215-240.

WORLD BANK (2005). World development report 2006. Equity and development. Washington, D.C.: World Bank.

WORLD BANK (2006). World development report 2007. Development and the next generation. Washington, D.C.: World Bank.

WORLD BANK (2007). World development indicators 2007. Washington, D.C.: World Bank. 